Ciência Florestal, Santa Maria, v. 21, n. 2, p. 303-313, abr.-jun., 2011

ISSN 0103-9954

\title{
MODELOS DE CRESCIMENTO EM DIÂMETRO DE ÁRVORES INDIVIDUAIS DE Araucaria angustifolia (Bertol.) Kuntze EM FLORESTA OMBRÓFILA MISTA
}

\section{INDIVIDUAL TREE DIAMETER GROWTH MODEL FOR Araucaria angustifolia (Bertol.) Kuntze IN MIXED OMBROPHYLOUS FOREST}

\author{
Tatiane Chassot $^{1}$ Frederico Dimas Fleig ${ }^{2}$ César Augusto Guimarães Finger ${ }^{3}$ Solon Jonas Longhi ${ }^{4}$
}

\section{RESUMO}

Este trabalho objetivou modelar o crescimento em diâmetro para árvores individuais de Araucaria angustifolia, ocorrentes na Floresta Ombrófila Mista no nordeste do Rio Grande do Sul. Para tanto, foram utilizadas remedições em intervalo de 7 anos de 251 indivíduos de araucária ocorrentes nas parcelas permanentes do Projeto PELD/CNPq. Foi construído um modelo de produção, tendo como variável dependente o diâmetro futuro, utilizando regressão e análise de covariância. Como variáveis independentes, foram utilizadas as características dimensionais e sociológicas dos indivíduos e os índices de concorrência independentes de distância. A variável mais correlacionada com o diâmetro futuro foi o diâmetro atual. Os índices de concorrência apresentaram alta correlação com a variável dependente, representando a pressão competitiva exercida sobre a árvore, mas não foram selecionados no modelo de regressão. Somente o diâmetro atual, multiplicado por um coeficiente, acrescido de um valor constante o qual diferiu de acordo com a posição sociológica do indivíduo, foi eficiente para predizer o diâmetro futuro. Foram construídos três variações do modelo de produção. Esses modelos apresentaram melhores estimativas para indivíduos de Araucaria angustifolia emergentes, no entanto, o seu emprego na prognose de crescimento de árvores individuais deve ser cauteloso por causa da magnitude dos erros de predição que apresentou.

Palavras-chave: concorrência; incremento diamétrico; pinheiro-brasileiro.

\begin{abstract}
This work aims to model the diameter growth for individual trees of Araucaria angustifolia, present in the Mixed Ombrophylous Forest in the northeast of Rio Grande do Sul. Thus, 251 araucaria individuals located on the permanent plots of the Project PELD - CNPq were remeasured after a period of 8 years. A yield model was built, having as dependent variable the future diameter, using regression and analysis of covariance. As independent variables, the dimensional and social characteristics of the individuals and distance independent competition indices were used. The most correlated variable with the future diameter was the current diameter. The competition indices showed high correlation with the dependent variable, representing the competitive pressure exerted on the tree, but they were not selected in the regression model. Only the current diameter, multiplied by a coefficient, plus a constant value which differed according to the sociological position of the individuals was effective to predict the future diameter. Three variations of the production model were built. The models showed better estimates for emerging individuals of
\end{abstract}

1. Engenheiro Florestal, Msc, Doutoranda do Programa de Pós-graduação em Engenharia Florestal, Centro de Ciências Rurais, Universidade Federal de Santa Maria, Av. Roraima, 1000, CEP 97105-900, Santa Maria (RS). tatianechassot@uffs.edu.br

2. Engenheiro Florestal, Dr., Professor Adjunto do Departamento de Ciências Florestais, Centro de Ciências Rurais, Universidade Federal de Santa Maria, Av. Roraima, 1000, CEP 97105-900, Santa Maria (RS). dimasfleig@uol.com.br

3. Engenheiro Florestal, Dr., Professor Associado do Departamento de Ciências Florestais, Centro de Ciências Rurais, Universidade Federal de Santa Maria, Av. Roraima, 1000, CEP 97105-900, Santa Maria (RS). caesar.finger@gmail.com

4. Engenheiro Florestal, Dr., Professor Titular do Departamento de Ciências Florestais, Centro de Ciências Rurais, Universidade Federal de Santa Maria, Av. Roraima, 1000, CEP 97105-900, Santa Maria (RS). longhi.solon@gmail.com

Recebido para publicação em 31/03/2009 e aceito em 26/08/2010 
Araucaria angustifolia, however, its use in the individual tree growth prognosis should be cautious due to the magnitude of the errors it presented.

Keywords: competition; diameter increase; Parana pine.

\section{INTRODUÇÃO}

Uma das maneiras de se obter conhecimento sobre o crescimento das espécies é utilizando modelos de crescimento, os quais possibilitam a predição de produção em tempos futuros, partindo-se das condições atuais.

Segundo Campos e Leite (2006), há uma extensa literatura no que diz respeito a modelos de crescimento de florestas homogêneas e equiâneas. Nessas florestas, o incremento é determinado em função das características médias das variáveis dimensionais, das árvores médias do povoamento e em função da idade. No entanto, esse tipo de modelo é simples e muitos não explicam diretamente a variação do tamanho das árvores dentro do povoamento e não são aplicáveis para florestas de múltiplas espécies com diferentes idades (VANCLAY, 1994).

Povoamentos inequiâneos possuem um elevado nível de complexidade e diversidade, com uma vasta gama de espécies, nas mais variadas alturas e tamanhos de copa, resultando em um perfil vertical irregular do povoamento. Além disso, cada espécie apresenta um conjunto de características singulares, como ritmo de crescimento, necessidades por nutrientes, luz e espaço para crescimento, em um período de desenvolvimento bem mais longo quando comparado a uma floresta pura (KIERMAN et al., 2008).

Assim, segundo Vanclay (1994), para determinar o crescimento e incremento de espécies que compõem as florestas nativas, deve-se lançar mão de técnicas de modelagem de crescimento considerando as árvores de forma individual.

Segundo Della-Flora (2001), esses modelos têm como vantagem o detalhamento da estrutura do povoamento, além de permitirem a análise de aspectos fisiológicos e de coexistência. Nesses modelos, não é mais o povoamento a unidade de prognose, mas, sim, cada árvore individual. Nas tabelas de produção usuais, a média das dimensões e totais por unidade de área será o somatório dos incrementos das árvores singulares.

Em função do tipo de índice de concorrência utilizado, os modelos de crescimento para árvores singulares foram divididos em dois principais grupos: os modelos espaciais, chamados de modelos dependentes de distância, nos quais são necessárias informações sobre a localização das árvores na floresta e os não espaciais, chamados de modelos independentes de distância. Os modelos não espaciais são mais simples e exigem menor levantamento de dados, pois não utilizam dados espaciais de localização (PORTÉ e BARTELINK, 2002; VANCLAY, 1994).

A modelagem do crescimento de árvores individuais vem sendo desenvolvida em especial em países europeus e norte-americanos. Conforme Hasenauer (1994), o primeiro modelo simulador de árvore singular foi desenvolvido por Newhan, no ano de 1964, para povoamentos puros. A partir de então, os pesquisadores passaram a trabalhar muito com modelagem, orientada em árvores singulares.

Para Araucaria angustifolia, já existem estudos para povoamentos homogêneos equiâneos e inequiâneos, como a tabela de produção desenvolvida por Schneider et al. (1992), e o estudo de Hess et al. (2009) que avaliaram o crescimento da araucária em função da idade. Porém, nesses estudos, realizados com objetivo de planejar a produção e realizar a prognose do crescimento, as estimativas foram baseadas em valores médios do povoamento.

Della-Flora (2001) formulou modelos de crescimento para Nectandra megapotamica Mez. e Ocotea puberula (Rich.) Nees, espécies da Floresta Estacional Decidual do Rio Grande do Sul. Mattos (2007) modelou o incremento periódico anual em área basal para Cabralea canjerana (Vell.) Mart., Cedrela fissilis (Vell.) e Cordia tricotoma (Vell.) Arrabida ex Steudel. As variáveis independentes dos modelos foram as características dimensionais individuais e sociológicas, de sítio e de concorrência.

$\mathrm{O}$ crescimento em diâmetro de árvores individuais pode ser expresso como incremento em diâmetro ou em área basal. Segundo Vanclay (1994), pesquisadores discutem a preferência da modelagem do incremento em área basal sobre a modelagem do incremento em diâmetro, já que o incremento em área basal supostamente seria mais correlacionado com o crescimento em volume atingido por uma árvore e também pelo fato de os modelos de incremento em área basal apresentarem maior coeficiente de determinação $\left(R^{2}\right)$. No entanto, segundo o autor, essa diferença na precisão do modelo é em virtude da estrutura do erro e implica em uma relação funcional, em vez de superioridade de um modelo sobre o outro. 
Outra decisão a ser tomada na modelagem é em relação à variável resposta, ou seja, modelar o diâmetro futuro ou o incremento em diâmetro. De acordo com Vanclay (1994), o aumento do diâmetro do tronco pode ser expresso como uma função de produção, a qual estima diâmetros futuros, ou como uma função de crescimento, que estima o incremento durante um período específico.

O crescimento de uma floresta depende tanto da produtividade potencial, dada pela qualidade de sítio, quanto pelo grau em que se aproveita essa potencialidade expressa pelo estoqueamento da floresta. De acordo com Prodan et al. (1997), o conceito de densidade está estritamente ligado às condições de concorrência, ocupação da superfície e fechamento do dossel.

Assim, a densidade pode ser utilizada como variável de decisão para intervenções silviculturais, como também em modelos simuladores de crescimento e na prognose de produção, nos quais é expressa por meio de índices de concorrência, que podem ou não levar em consideração a distância entre a árvore concorrida e a concorrente.

Os índices de concorrência ou competição expressamuma estimativa do espaço horizontal ocupado por cada árvore-amostra em relação a suas vizinhas. Nos índices independentes de distância, a concorrência é medida por meio da relação das dimensões da árvore sujeito com as variáveis do povoamento, como número de árvores por hectare, área basal por hectare e altura média.

Este trabalho objetivou construir um modelo de crescimento diamétrico para árvores individuais da espécie Araucaria angustifolia como função de suas características dimensionais individuais, sociológicas e da concorrência.

\section{MATERIAL E MÉTODO}

\section{Caracterização e descrição da área de estudo}

AFloresta Ombrófila Mista, Mata de Araucária ou de Pinheiro-brasileiro tem como característica o predomínio da Araucaria angustifolia. Além dessa espécie, existem muitas outras que juntas formam comunidades interativas e diferenciadas em florística, estrutura e organização ecológica. A riqueza dessa floresta pode ser encontrada nos seus componentes arbóreos, no subdossel ou nas copas que formam dosséis irregulares, atingindo assim, níveis elevados de biodiversidade, apesar de sua aparente simplicidade estrutural (SANQUETTA, 2005).

A Floresta Ombrófila Mista pertencente à
Floresta Nacional (FLONA) de São Francisco de Paula ultimamente tem sido alvo de muitos estudos, pois nela está locado um conjunto de parcelas integrantes do Projeto Ecológico de Longa Duração (PELD/CNPq) "Conservação e Manejo Sustentável de Ecossistemas Florestais - Bioma Araucária e suas Transições".

A FLONA localiza-se no Distrito de Rincão dos Kroeff, no município de São Francisco de Paula, nordeste do Rio Grande do Sul, a $27 \mathrm{~km}$ da sede do Município, a uma altitude de $930 \mathrm{~m}$ acima do nível do mar. A temperatura média anual é de $14,5^{\circ} \mathrm{C}$ e a precipitação média de $2.500 \mathrm{~mm} /$ anuais (NIMER, 1990). O clima caracteriza-se, segundo a classificação climática de Köppen, como "Cfb" (mesotérmico subtropical, com período de temperado; úmido com precipitação uniformemente distribuída durante $\mathrm{O}$ ano; verão brando, característico do Planalto do RS) (MORENO, 1961).

O solo é do tipo Cambissolo Húmico Alumínico Típico - CHa 1 (STRECK et al., 2008) e apresenta como características principais o horizonte $\mathrm{A}$ húmico de elevada acumulação de matéria orgânica e o horizonte B incipiente, porém menos ricos em matéria orgânica (NARVAES, 2004).

De uma área total de 1.606,69 hectares da FLONA, 901,9 ha correspondem à Floresta Ombrófila Mista (56,1\%) (IBDF/FATEC, 1989).

As espécies pertencentes à floresta em estudo apresentaram um diâmetro médio de 21,95 cm, com diâmetro máximo de 143,63 cm pertencente a um indivíduo de Arancaria angustifolia. A altura total média e a altura comercial média estimada dos indivíduos foram de $13,12 \mathrm{~m} \mathrm{e} 7,79 \mathrm{~m}$, respectivamente, a área basal e o volume comercial foram de $45,69 \mathrm{~m}^{2} /$ ha e $337,72 \mathrm{~m}^{3} /$ ha respectivamente. Segundo Ribeiro (2004), autor que classificou a comunidade arbórea das parcelas em estudo, todos esses valores foram maiores que os encontrados pelo Inventário Florestal Contínuo do Rio Grande do Sul, em 2002, comprovando que a Floresta Ombrófila Mista da FLONA de São Francisco de Paula apresenta maior estoque de madeira/ha em relação às outras formações florestais do Estado.

A Araucaria angustifolia (Bertol.) Kuntze, espécie pertencente à família Araucariaceae, popularmente conhecida por araucária, pinheiro-doparaná, pinheiro-brasileiro, é uma típica conífera brasileira, de grande porte, tronco reto e quase cilíndrico, com altura variando entre 10 e $35 \mathrm{~m}$, fuste com até 20 $\mathrm{m}$ ou mais, e diâmetro à altura do peito acima de $50 \mathrm{~cm}$ quando adulta, alcançando melhor desenvolvimento a partir dos 30 anos de idade (AQUINO, 2005). 
Sua madeira é bastante utilizada para forros, molduras, ripas, caixotaria, brinquedos, utensílios domésticos, entre outros. Foi amplamente cultivada no sul do Brasil para produção de madeira, pasta celulósica, construção civil e laminação. O fruto é comestível e de grande importância para a alimentação humana e da fauna, tanto de aves, como de mamíferos. É também uma espécie ornamental, podendo ser empregada no paisagismo (LORENZI, 1998).

De acordo com estudo realizado por Ribeiro (2004) na Floresta Ombrófila Mista da Floresta Nacional de São Francisco de Paula, a Araucaria angustifolia destacou-se na fisionomia da floresta por apresentar alta densidade e dominância, considerada, assim, a espécie mais importante, representando $15,56 \%$ do Valor de Importância e 22,49\% do Valor de Cobertura.

\section{Obtenção de dados}

As unidades amostrais utilizadas na obtenção de dados neste trabalho possuem um tamanho de $100 \mathrm{x}$ $100 \mathrm{~m}$ (área de 1 ha), subdivididas em dez faixas (100 x $10 \mathrm{~m}$ ) divididas em dez subunidades (10 x $10 \mathrm{~m})$.

Em todas as dez unidades amostrais foram levantados por equipe do projeto PELD, anualmente, desde 0 ano de 2000 as seguintes variáveis: identificação das espécies; circunferência à altura do peito (cap); altura total e comercial; posição sociológica: 1 - árvore emergente; 2 - árvore intermediária; 3 - subdossel; 4 - árvore suprimida; tendência à valorização: 1 crescimento promissor, mudança ascendente na posição sociológica; 2 - crescimento médio, mudança lenta na posição sociológica; 3 - crescimento insignificante, tendência a permanecer na mesma posição sociológica; classe de copa: 1 - copa longa, comprimento maior que $1 / 2$ da altura total da árvore; 2 - copa média, comprimento da copa entre $1 / 2$ e $1 / 4$ da altura total da árvore; 3 - copa curta, comprimento da copa inferior a $1 / 4$ da altura total da árvore; 4 - copa danificada ou quebrada. Assim, para este estudo, foram utilizadas as medições de sete anos.

Essas características foram avaliadas para a totalidade das árvores pertencentes às parcelas. Essas árvores foram identificadas com etiqueta de alumínio fixadas com prego, dispostas a $0,30 \mathrm{~m}$ da base da árvore e a altura do diâmetro à altura do peito (DAP), no tronco, foi demarcada com tinta.

\section{Procedimento de cálculo - Modelagem}

Após a aquisição dos dados, foi feita uma revisão destes, excluindo dados inconsistentes, árvores mortas, indivíduos que não tinham 8 anos de medição e os que apresentavam a copa quebrada.

As variáveis utilizadas para modelagem foram o DAP e a área basal medidos em 2000 e em 2007, a altura total, a relação hipsométrica $(\mathrm{h} / \mathrm{d})$, a posição sociológica, a tendência à valorização, a classe de copa e os índices de concorrência independentes de distância, calculados para o ano de 2007.

Foi construído um modelo de produção, tendo como variável dependente o DAP futuro, utilizando-se o software Statistical Package for the Social Sciences (SPSS) por meio da análise de regressão com o procedimento Stepwise, análise de correlação, análise de correlação parcial e análise de covariância.

O DAP futuro foi modelado em função das características dimensionais das árvores, ou seja, pelo dap (diâmetro à altura do peito), altura, área basal, relação $\mathrm{h} / \mathrm{d}$, pelas características sociológicas como a posição sociológica, classe de copa, tendência de valorização e pela concorrência. As características de sítio não foram utilizadas para a modelagem, pois não estiveram incluídas no levantamento dos dados.

Para quantificar a concorrência, foram calculados os índices apresentados na Tabela 1.

TABELA 1: Índices de concorrência independentes de distância utilizados na modelagem do crescimento em diâmetro de Araucaria angustifolia.

TABLE 1: Distance independent competition indices used for modeling the diameter increment of Araucaria angustifolia.

\begin{tabular}{lc}
$\begin{array}{l}\text { Índice de Glover e Hool (1979) } \\
\text { apud Prodan et at. (1997) }\end{array}$ & $I_{G H}=\frac{d_{i}^{2}}{\bar{d}^{2}}$ \\
\hline Índice de Glover e Hool (1979) & $I_{1}=\frac{h_{i}}{\bar{h}}$ \\
\hline Índice de Glover e Hool (1979) & $I_{2}=\frac{d_{i}^{2} \cdot h_{i}}{\bar{d}^{2} \cdot \bar{h}}$ \\
\hline $\begin{array}{l}\text { Índice de BAL } \\
\text { (Basal Area Larger) }\end{array}$ & $B A L=\frac{G_{m e ́ d i o}}{g_{i}}$ \\
\hline $\begin{array}{l}\text { Índice de BAL } \\
\text { (Basal Area Larger) }\end{array}$ & $B A L 1=\frac{G_{\text {maior }}}{G / h a}$ \\
\hline
\end{tabular}

Em que: $d_{i}$ : diâmetro da árvore considerada, em centímetros; $\overline{\mathrm{d}}$ : diâmetro médio do povoamento, em centímetros; $h_{\mathrm{i}}$ : altura da árvore considerada, em metros; $\overline{\mathrm{h}}$ : altura média do povoamento, em metros; $\mathrm{G}_{\text {médio }}$ : área basal média por unidade amostral, em $\mathrm{m}^{2} ; \mathrm{g}_{\mathrm{i}}$ : área basal da árvore considerada, em $\mathrm{m}^{2} ; \mathrm{G}_{\text {maior }}$ : área basal das árvores com dap maior que o dap da árvore considerada, por hectare, em $\mathrm{m}^{2}$; G/ha: área basal por hectare. 
Para o cálculo dos índices de concorrência, foram utilizados diferentes tamanhos de unidades amostrais (área total de 1 ha, subunidade de $10 \times 10$ $\mathrm{m}$, e $50 \times 50 \mathrm{~m}$ ).

Após a obtenção dos modelos de produção para Araucaria angustifolia, foram analisadas a distribuição dos resíduos do modelo.

\section{RESULTADOS E DISCUSSÕES}

\section{Descrição das variáveis estudadas}

Após depuração e validação dos dados, foram utilizadas, para este estudo, informações dimensionais, sociológicas e de concorrência de um total de 251 indivíduos de Araucaria angustifolia.

Entre as variáveis de classificação, a classe de copa não apresentava grande variação, pois a maioria das árvores apresentava classe de copa 3 , inclusive com a média desse valor sendo igual a 2,97. Além disso, a classe de copa bem como a tendência de valorização são fatores cuja determinação é bastante subjetiva. Já a posição sociológica pode ser mais facilmente determinada e ter uma relação estreita com variáveis de concorrência que utilizam a altura do indivíduo considerado e da população.
Os dados estatísticos levantados e calculados para os indivíduos da espécie Araucaria angustifolia, separados por posição sociológica, podem ser observados na Tabela 2.

$\mathrm{O}$ valor mínimo do índice IGH (Índice de Glover e Hool), calculado para os diferentes tamanhos de unidade amostral, não variou. Já o valor máximo desse índice variou de 19,60 a 44,30, para uma unidade amostral de $10 \times 10 \mathrm{~m}$ e $50 \mathrm{x}$ $50 \mathrm{~m}$, respectivamente, para árvores emergentes. Essa variação dos valores máximos de IGH deu-se, possivelmente, em função da constante mudança dos valores de diâmetro médio para a unidade amostral de $10 \times 10 \mathrm{~m}$, quando comparado aos diâmetros médios para as unidades amostrais de 1 hectare e de $50 \times 50 \mathrm{~m}$, para as quais o diâmetro médio variava nenhuma e quatro vezes, respectivamente.

Para o índice de Basal Area Larger (BAL), não foram observadas variações dos valores entre os tamanhos de unidade amostral, quando calculados partindo da relação $\mathrm{G}_{\text {médio }} / \mathrm{g}_{\mathrm{i}}$. No entanto, para BAL, calculado partindo da relação com as árvores mais grossas que a árvore de referência, houve uma diferença para a média e o valor máximo da área basal.

TABELA 2: Estatística descritiva para árvores selecionadas de Araucaria angustifolia nas parcelas do PELD - FLONA - SFP, RS, separados por posição sociológica.

TABLE 2: Descriptive statistics for selected trees of Araucaria angustifolia in plots of PELD - FLONA - SFP, RS, separated by sociological position.

\begin{tabular}{|c|c|c|c|c|c|c|c|c|c|c|c|c|c|c|c|c|}
\hline & & & & Emergen & & & & & nterme & liária & & & & Subdo & ssel & \\
\hline & Variáveis & $\mathrm{N}$ & Mínimo & Média & Máxir & $\begin{array}{l}\text { Desvio } \\
\text { Padrão }\end{array}$ & $\mathrm{N}$ & Mínime & Média & Máxim & $\begin{array}{l}\text { Desvio } \\
\text { Padrão }\end{array}$ & $\mathrm{N}$ & Mínimo & Média & Máximo & $\begin{array}{l}\text { Desvio } \\
\text { Padrão }\end{array}$ \\
\hline & Inc. dap & 126 & 0,70 & 2,28 & 6,59 & 1,15 & 107 & 0,73 & 2,08 & 5,25 & 1,17 & 18 & 0,70 & 1,08 & 1,97 & 0,37 \\
\hline & dap2007 & 126 & 22,79 & 66,15 & 145,44 & 17,99 & 107 & 12,61 & 33,98 & 72,42 & 12,78 & 18 & 10,73 & 16,73 & 28,11 & 5,20 \\
\hline & $0-25$ & 0 & 0,00 & 0,00 & 0,00 & 0,00 & 25 & 12,61 & 19,25 & 24,03 & 3,51 & 16 & 10,73 & 15,35 & 23,08 & 3,52 \\
\hline classe & $25-50$ & 15 & 22,79 & 37,18 & 47,62 & 6,75 & 70 & 25,08 & 34,89 & 49,37 & 6,91 & 2 & 27,44 & 27,78 & 28,11 & 0,47 \\
\hline de & $50-75$ & 77 & 50,77 & 62,13 & 74,96 & 6,61 & 12 & 50,67 & 59,37 & 72,42 & 6,72 & 0 & 0,00 & 0,00 & 0,00 & 0,00 \\
\hline dap & $75-100$ & 30 & 75,34 & 83,85 & 93,74 & 5,68 & 0 & 0,00 & 0,00 & 0,00 & 0,00 & 0 & 0,00 & 0,00 & 0,00 & 0,00 \\
\hline & acima de 100 & 4 & 104,88 & 119,28 & 145,44 & 18,71 & 0 & 0,00 & 0,00 & 0,00 & 0,00 & 0 & 0,00 & 0,00 & 0,00 & 0,00 \\
\hline & ht & 126 & 18,30 & 23,65 & 32,30 & 2,65 & 107 & 11,10 & 19,02 & 24,10 & 2,60 & 18 & 10,30 & 13,18 & 17,20 & 1,82 \\
\hline & hd & 126 & 0,20 & 0,38 & 0,87 & 0,10 & 107 & 0,26 & 0,62 & 1,20 & 0,19 & 18 & 0,46 & 0,83 & 1,13 & 0,18 \\
\hline & I1 & 126 & 1,50 & 1,76 & 2,90 & 0,34 & 107 & 1,00 & 1,22 & 1,50 & 0,14 & 18 & 0,70 & 0,88 & 1,00 & 0,12 \\
\hline & $\mathrm{I} 2$ & 126 & 2,00 & 13,23 & 33,20 & 6,63 & 107 & 0,30 & 3,04 & 14,70 & 2,67 & 18 & 0,20 & 0,55 & 1,50 & 0,36 \\
\hline & IGHt & 126 & 1,10 & 9,62 & 42,10 & 5,46 & 107 & 0,30 & 2,69 & 11,70 & 2,13 & 18 & 0,20 & 0,62 & 1,60 & 0,40 \\
\hline & IGH10 & 126 & 1,20 & 7,51 & 19,60 & 3,48 & 107 & 0,30 & 2,37 & 9,80 & 1,90 & 18 & 0,20 & 0,61 & 1,50 & 0,35 \\
\hline & IGH50 & 126 & 1,20 & 9,62 & 44,30 & 5,64 & 107 & 0,20 & 2,60 & 10,60 & 2,01 & 18 & 0,20 & 0,62 & 1,70 & 0,42 \\
\hline & BAL10 & 126 & 0,10 & 0,25 & 0,90 & 0,12 & 107 & 0,10 & 1,03 & 6,70 & 0,94 & 18 & 0,70 & 2,85 & 6,20 & 1,43 \\
\hline & BAL50 & 126 & 0,00 & 0,22 & 1,20 & 0,17 & 107 & 0,10 & 1,01 & 5,70 & 0,99 & 18 & 0,90 & 3,29 & 6,50 & 1,65 \\
\hline & BALt & 126 & 0,00 & 0,22 & 1,40 & 0,18 & 107 & 0,10 & 0,99 & 5,40 & 0,91 & 18 & 0,90 & 3,36 & 6,90 & 1,78 \\
\hline & BAL1 & 126 & 0,00 & 0,06 & 0,70 & 0,16 & 107 & 0,00 & 0,49 & 1,00 & 0,31 & 18 & 0,20 & 0,79 & 1,00 & 0,21 \\
\hline
\end{tabular}


A maior parte dos indivíduos selecionados pertenceu à posição sociológica emergente, apresentou crescimento promissor e copa curta, não tendo sido analisado nenhum indivíduo de copa longa.

\section{Incremento em diâmetro para Araucaria angustifolia}

O crescimento das árvores é decorrente da atividade meristemática e resulta na elongação e no aumento da espessura das raízes, troncos e galhos, ocasionando mudanças no peso, tamanho e forma das árvores. Esse crescimento não é constante durante toda a vida de uma árvore. Para a maioria das espécies, observa-se uma diminuição do incremento em DAP (ENCINAS et al., 2005). Para os indivíduos de araucária estudados observou-se tal diminuição.

Os indivíduos do subdossel apresentaram um incremento médio, para um período de 7 anos, 1 $\mathrm{cm}$ menor que as árvores intermediárias e estas 0,2 $\mathrm{cm}$ menores que as emergentes.

Somente árvores emergentes com diâmetros acima de $75 \mathrm{~cm}$ atingiram valores decrescentes de incremento, mas esse fato pode estar relacionado em grande parte à escassa amostragem.

Fazendo uma análise de correlação entre as variáveis dimensionais individuais e de concorrência para os indivíduos de Araucaria angustifolia, observou-se que o incremento em diâmetro apresentou baixa correlação com as demais variáveis e, na maioria das vezes, essa correlação não foi significativa, como pode ser observado na Tabela 3.

A variável melhor correlacionada com o diâmetro do ano de 2007 foi o diâmetro de 2000, apresentando uma correlação significativa de 0,99. Isso mostra que a variável diâmetro pode ser usada

TABELA 3: Correlação de Pearson e probabilidades para as variáveis dimensionais e de concorrência para Araucaria angustifolia.

TABLE 3: Pearson correlation and probabilities of dimension variables and concurrence for Araucaria angustifolia.

\begin{tabular}{|c|c|c|c|c|c|c|c|c|c|c|c|c|c|c|c|c|c|c|c|}
\hline Variáveis & NCDAP & DAP2000 & DAP2007 & g2000 & g2007 & HD & HT & BAL1 & BAL10 & BAL50 & BALT & I1 & $\mathrm{I} 2$ & IGH10 & IGH50 & IGHT & $\mathrm{CC}$ & PS & TV \\
\hline INCDAP & 1 & 0,06 & 0,11 & 0,02 & 0,06 & $-0,15$ & 0,17 & $-0,20$ & $-0,16$ & $-0,23$ & $-0,23$ & 0,04 & 0,03 & 0,08 & 0,05 & 0,06 & $-0,13$ & $-0,22$ & $-0,07$ \\
\hline DAP2000 & 0,36 & 1 & 0,99 & 0,96 & 0,96 & $-0,88$ & 0,80 & $-0,72$ & $-0,62$ & $-0,66$ & $-0,66$ & 0,61 & 0,81 & 0,85 & 0,95 &, 96 & 0,04 & $-0,74$ & $-0,05$ \\
\hline DAP2007 & 0,09 & $\mathbf{0 , 0 0}$ & 1 & 0,95 & 0,96 & $-0,88$ & 0,80 & $-0,73$ & $-0,63$ & $-0,67$ & $-0,67$ & 0,61 & 0,81 & 0,85 & 0,95 & 0,96 & 0,03 & $-0,75$ & $-0,06$ \\
\hline g2000 & 0,76 & $\mathbf{0 , 0 0}$ & $\mathbf{0 , 0 0}$ & 1 & 1,00 & $-0,74$ & 0,71 & $-0,60$ & $-0,48$ & $-0,50$ & $-0,50$ & 0,50 & 0,75 & 0,79 & 0,99 & 1,00 & 0,02 & $-0,63$ & $-0,03$ \\
\hline g2007 & 0,34 & $\mathbf{0 , 0 0}$ & $\mathbf{0 , 0 0}$ & $\mathbf{0 , 0 0}$ & 1 & $-0,75$ & 0,71 & $-0,61$ & $-0,49$ & $-0,51$ & $-0,51$ & 0,50 & 0,76 & 0,80 & 0,99 & 1,00 & 0,02 & $-0,64$ & $-0,03$ \\
\hline HD & 0,02 & $\mathbf{0 , 0 0}$ & $\mathbf{0 , 0 0}$ & $\mathbf{0 , 0 0}$ & $\mathbf{0 , 0 0}$ & 1 & $-0,67$ & 0,76 & 0,74 & 0,83 & 0,82 & $-0,56$ & $-0,70$ & $-0,75$ & $-0,74$ & $-0,75$ & $-0,04$ & 0,70 & 0,12 \\
\hline HT & $\mathbf{0 , 0 0}$ & $\mathbf{0 , 0 0}$ & $\mathbf{0 , 0 0}$ & $\mathbf{0 , 0 0}$ & $\mathbf{0 , 0 0}$ & $\mathbf{0 , 0 0}$ & 1 & $-0,66$ & $-0,64$ & $-0,72$ & $-0,73$ & 0,68 & 0,67 & 0,65 & 0,70 & 0,71 & 0,07 & $-0,77$ & $-0,09$ \\
\hline BAL1 & $\mathbf{0 , 0 0}$ & $\mathbf{0 , 0 0}$ & $\mathbf{0 , 0 0}$ & $\mathbf{0 , 0 0}$ & $\mathbf{0 , 0 0}$ & $\mathbf{0 , 0 0}$ & $\mathbf{0 , 0 0}$ & 1 & 0,76 & 0,67 & 0,66 & $-0,63$ & $-0,67$ & $-0,70$ & $-0,60$ & $-0,61$ & $-0,06$ & 0,72 & 0,07 \\
\hline BAL10 & $\mathbf{0 , 0 1}$ & $\mathbf{0 , 0 0}$ & $\mathbf{0 , 0 0}$ & $\mathbf{0 , 0 0}$ & $\mathbf{0 , 0 0}$ & $\mathbf{0 , 0 0}$ & $\mathbf{0 , 0 0}$ & $\mathbf{0 , 0 0}$ & 1 & 0,81 & 0,79 & $-0,55$ & $-0,54$ & $-0,58$ & $-0,48$ & $-0,49$ & $-0,18$ & 0,66 & 0,15 \\
\hline BAL50 & $\mathbf{0 , 0 0}$ & $\mathbf{0 , 0 0}$ & $\mathbf{0 , 0 0}$ & $\mathbf{0 , 0 0}$ & $\mathbf{0 , 0 0}$ & $\mathbf{0 , 0 0}$ & $\mathbf{0 , 0 0}$ & $\mathbf{0 , 0 0}$ & $\mathbf{0 , 0 0}$ & 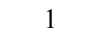 & 0,99 & $-0,54$ & $-0,51$ & $-0,54$ & $-0,51$ & $-0,51$ & $-0,11$ & 0,66 & 0,18 \\
\hline BALT & $\mathbf{0 , 0 0}$ & $\mathbf{0 , 0 0}$ & $\mathbf{0 , 0 0}$ & 0,00 & $\mathbf{0 , 0 0}$ & $\mathbf{0 , 0 0}$ & $\mathbf{0 , 0 0}$ & $\mathbf{0 , 0 0}$ & $\mathbf{0 , 0 0}$ & $\mathbf{0 , 0 0}$ & 1 & $-0,54$ & $-0,51$ & $-0,54$ & $-0,51$ & $-0,52$ & $-0,10$ & 0,67 & 0,19 \\
\hline I1 & 0,51 & $\mathbf{0 , 0 0}$ & $\mathbf{0 , 0 0}$ & $\mathbf{0 , 0 0}$ & $\mathbf{0 , 0 0}$ & $\mathbf{0 , 0 0}$ & $\mathbf{0 , 0 0}$ & $\mathbf{0 , 0 0}$ & $\mathbf{0 , 0 0}$ & $\mathbf{0 , 0 0}$ & $\mathbf{0 , 0 0}$ & 1 & 0,74 & 0,59 & 0,51 & 0,50 & 0,05 & $-0,76$ & 0,25 \\
\hline I2 & 0,60 & $\mathbf{0 , 0 0}$ & $\mathbf{0 , 0 0}$ & 0,00 & $\mathbf{0 , 0 0}$ & $\mathbf{0 , 0 0}$ & $\mathbf{0 , 0 0}$ & $\mathbf{0 , 0 0}$ & $\mathbf{0 , 0 0}$ & $\mathbf{0 , 0 0}$ & $\mathbf{0 , 0 0}$ & $\mathbf{0 , 0 0}$ & 1 & 0,96 & 0,76 & 0,76 & 0,06 & $-0,70$ & 0,08 \\
\hline IGH10 & 0,23 & $\mathbf{0 , 0 0}$ & $\mathbf{0 , 0 0}$ & $\mathbf{0 , 0 0}$ & $\mathbf{0 , 0 0}$ & $\mathbf{0 , 0 0}$ & $\mathbf{0 , 0 0}$ & $\mathbf{0 , 0 0}$ & $\mathbf{0 , 0 0}$ & $\mathbf{0 , 0 0}$ & $\mathbf{0 , 0 0}$ & $\mathbf{0 , 0 0}$ & $\mathbf{0 , 0 0}$ & 1 & 0,80 & 0,80 & 0,06 & $-0,68$ & $-0,03$ \\
\hline IGH50 & 0,39 & $\mathbf{0 , 0 0}$ & $\mathbf{0 , 0 0}$ & $\mathbf{0 , 0 0}$ & $\mathbf{0 , 0 0}$ & $\mathbf{0 , 0 0}$ & $\mathbf{0 , 0 0}$ & $\mathbf{0 , 0 0}$ & $\mathbf{0 , 0 0}$ & $\mathbf{0 , 0 0}$ & $\mathbf{0 , 0 0}$ & $\mathbf{0 , 0 0}$ & $\mathbf{0 , 0 0}$ & 0,00 & 1 & 0,99 & 0,00 & $-0,64$ & 0,00 \\
\hline IGHT & 0,37 & $\mathbf{0 , 0 0}$ & $\mathbf{0 , 0 0}$ & 0,00 & $\mathbf{0 , 0 0}$ & $\mathbf{0 , 0 0}$ & $\mathbf{0 , 0 0}$ & $\mathbf{0 , 0 0}$ & $\mathbf{0 , 0 0}$ & $\mathbf{0 , 0 0}$ & $\mathbf{0 , 0 0}$ & $\mathbf{0 , 0 0}$ & $\mathbf{0 , 0 0}$ & 0,00 & $\mathbf{0 , 0 0}$ & 1 & 0,01 & $-0,64$ & $-0,02$ \\
\hline $\mathrm{CC}$ & 0,04 & $\mathbf{0 , 5 7}$ & 0,65 & 0,72 & 0,79 & $\mathbf{0 , 5 5}$ & 0,25 & 0,32 & 0,00 & 0,08 & 0,13 & 0,41 & 0,35 & 0,38 & 0,95 & $\mathbf{0 , 8 5}$ & 1 & 0,02 & $-0,18$ \\
\hline PS & $\mathbf{0 , 0 0}$ & $\mathbf{0 , 0 0}$ & $\mathbf{0 , 0 0}$ & $\mathbf{0 , 0 0}$ & $\mathbf{0 , 0 0}$ & $\mathbf{0 , 0 0}$ & $\mathbf{0 , 0 0}$ & $\mathbf{0 , 0 0}$ & $\mathbf{0 , 0 0}$ & $\mathbf{0 , 0 0}$ & $\mathbf{0 , 0 0}$ & $\mathbf{0 , 0 0}$ & $\mathbf{0 , 0 0}$ & 0,00 & $\mathbf{0 , 0 0}$ & $\mathbf{0 , 0 0}$ & 0,75 & 1 & $-0,06$ \\
\hline TV & $\mathbf{0 , 3 0}$ & 0,39 & 0,36 & 0,65 & $\mathbf{0 , 6 0}$ & $\mathbf{0 , 0 7}$ & 0,15 & 0,29 & $\mathbf{0 , 0 1}$ & $\mathbf{0 , 0 1}$ & $\mathbf{0 , 0 0}$ & $\mathbf{0 , 0 0}$ & 0,21 & 0,60 & 0,96 & 0,77 & $\mathbf{0 , 0 0}$ & $\mathbf{0 , 3 2}$ & 1 \\
\hline
\end{tabular}

Em que: INCDAP: incremento em diâmetro; DAP2000: diâmetro no ano de 2000; DAP2007: diâmetro no ano de 2007; g2000: área basal no ano de 2000; g2007: área basal no ano de 2007; HD: relação hipsométrica; HT: altura total; BAL1: índice de BAL calculado somente com as árvores mais grossas que a árvore sujeito; BAL10: índice de BAL calculado para uma área de 10×10m; BAL50: índice de BAL calculado para uma área de 50x50m; BALT: índice de BAL calculado para uma área de 1ha; I1: índice de Glover e Hool calculado a partir da relação das alturas; I2: índice de Glover e Hool calculado partindo da relação das alturas e diâmetros; IGH10: índice de Glover e Hool calculado para uma área de 10x10m; IGH50: índice de Glover e Hool calculado para uma área de 50x50m; IGHt: índice de Glover e Hool calculado para uma área de 1ha; CC: classe de copa; PS: posição sociológica; TV: tendência de valorização; Valores em negrito representam as probabilidades.

Ci. Fl., v. 21, n. 2, abr.-jun., 2011 
para modelagem de crescimento, em substituição à idade, variável utilizada para povoamentos equiâneos, sob a hipótese de que, quanto maior o diâmetro da árvore, maior é a sua idade, já que, em florestas nativas, a idade é de difícil determinação (STERBA, 1992).

Todas as variáveis apresentaram uma correlação significativa com a variável diâmetro, exceto classe de copa e tendência de valorização.

Para todos os tamanhos de unidade amostral utilizados, o diâmetro apresentou uma correlação negativa com os índices de concorrência de BAL, ou seja, quanto menor os valores desses índices, maior o diâmetro da árvore. Isso porque, quanto menor o índice de BAL, menor é a concorrência sob a qual a árvore está submetida, e consequentemente, apresenta maior diâmetro.

Para os índices de concorrência de Glover e Hool, o diâmetro apresentou uma correlação positiva, ou seja, quanto maiores os valores desses índices, maior o diâmetro da árvore. E também, quanto maior o valor desses índices, sob menor concorrência a árvore está submetida, e assim, apresenta maior diâmetro. O que demonstra que ambos os índices devam expressar a pressão competitiva a que uma árvore está submetida.

O diâmetro apresentou também uma correlação negativa para posição sociológica. Ou seja, árvores dominantes têm maior diâmetro que as árvores intermediárias ou do subdossel.

Observou-se também que a posição sociológica apresentou correlação significativa com todas as demais variáveis, excetuando-se a classe de copa, destacando a correlação da posição sociológica com os índices de concorrência de BAL e Glover e Hool.

Correlação negativa também foi observada para a relação altura diâmetro, ou seja, quanto maior essa relação, menor é o diâmetro da árvore.

Para as demais variáveis, $g_{\text {atual }}$ e altura total, a correlação foi positiva.

Controlando o efeito do diâmetro atual, verificou-se que a área basal, os diferentes índices de Glover e Hool e a tendência de valorização não se correlacionaram significativamente com o diâmetro futuro. Contudo, a relação hd, ht, classe de copa, posição sociológica e índices de BAL apresentaram correlação negativa, explicando menos de 30\%, como pode ser observado na Tabela 4.

Entre as variáveis de correlação negativa, destacou-se a posição sociológica a qual apresentou o maior valor, indicando que as árvores de araucária possivelmente apresentam padrão de crescimento diferenciado de acordo com sua posição sociológica.

Modelo de produção para Araucaria angustifolia

Por meio da análise de covariância, foi possível identificar que os indivíduos de Araucaria angustifolia apresentaram comportamento diferente em relação à posição sociológica que ocupam na floresta.

A variável posição sociológica foi significativa, sendo que os coeficientes variaram em função desta. Para indivíduos do subdossel o valor do coeficiente $\mathrm{b}_{0}$ foi de 1,241 , para indivíduos intermediários e emergentes foi acrescido, respectivamente, 1,159 e 1,690 (Tabela 5).

Assim, separando-se os indivíduos por posição sociológica, foram recalculados os coeficientes de cada modelo e foi possível obter modelos de predição em diâmetro com alta precisão, como pode ser observado na Tabela 6 .

De acordo com esses modelos, o diâmetro futuro é uma função do diâmetro atual, multiplicado por um coeficiente, e acrescido de um valor constante.

Estatisticamente, os valores de $b_{1}$ não diferiram de uma posição sociológica para outra. Assim, as retas têm a mesma inclinação, diferindo apenas o valor do intercepto, como pode ser observado na Figura 1. Essa Figura foi truncada aos $35 \mathrm{~cm}$ de diâmetro para que fosse possível a visualização das diferenças de níveis entre as curvas, pois para a amplitude total dos dados, a escala resultou grande em demasia, dificultando essa visualização.

Para um mesmo diâmetro, árvores emergentes apresentaram maior incremento em diâmetro em relação às intermediárias e subdossel.

TABELA 4: Correlações parciais e probabilidades entre as variáveis, com controle para o diâmetro.

TABLE 4: Partial correlation and probabilities among variables, with diameter control.

\begin{tabular}{llccccccccccccccc}
\hline & & g2000 & hd & ht & BAL1 & BAL10 & BAL50 & BALt & I1 & I2 & IGH10 IGH50 & IGHt & CC & P S & TV \\
\hline dap2007 & Correlação parcial & $-0,12$ & $-0,20$ & 0,21 & $-0,23$ & $-0,16$ & $-0,25$ & $-0,25$ & 0,01 & $-0,02$ & 0,05 & 0,00 & 0,01 & $-0,14$ & $-0,27$ & $-0,06$ \\
& Probabilidade & 0,52 & 0,01 & 0,01 & 0,00 & 0,00 & 0,00 & 0,00 & 0,90 & 0,71 & 0,42 & 0,97 & 0,93 & 0,03 & 0,00 & 0,33 \\
\hline
\end{tabular}


TABELA 5: Análise de covariância da função dap $p_{\text {futuro }}=\mathrm{b}_{0}+\mathrm{b}_{1}$ dap $_{\text {atual }}$ em função da posição sociológica dos indivíduos de Araucaria angustifolia.

TABLE 5: Analysis of covariance of function dap $p_{\text {futuro }}=\mathrm{b}_{0}+\mathrm{b}_{1}$ dap $\mathrm{p}_{\text {atual }}$ according to the sociological position of individuals of Araucaria angustifolia.

\begin{tabular}{|c|c|c|c|c|c|}
\hline Fonte de Variação & GL & SQ Tipo III & QM & $\mathrm{F}$ & Prob $>F$ \\
\hline Modelo & 3 & 137871,2 & 45957,052 & 36932,779 & 0,000 \\
\hline b0 & 1 & 152,812 & 152,812 & 122,805 & 0,000 \\
\hline $\mathrm{DAP}_{2000}$ & 1 & 57932,948 & 57932,948 & 46557,050 & 0,000 \\
\hline PS & 2 & 27,825 & 13,913 & 11,81 & 0,000 \\
\hline Erro & 247 & 307,353 & 1,244 & & \\
\hline Total & 250 & 738149,3 & & & \\
\hline \multicolumn{6}{|l|}{$\mathrm{R}^{2}$ ajus $=0,998$} \\
\hline Variável dependente & Parâmetros & Coeficientes & Erro-padrão & $\mathrm{t}$ & Prob $>t$ \\
\hline \multirow[t]{5}{*}{$\overline{\mathrm{DAP}}_{2007}$} & $\mathrm{~b}_{0}$ & 1,241 & 0,273 & 4,555 & 0,000 \\
\hline & dap2000 & 0,990 & 0,005 & 215,771 & 0,000 \\
\hline & Emergente & 1,690 & 0,358 & 4,726 & 0,000 \\
\hline & Intermediária & 1,159 & 0,294 & 3,945 & 0,000 \\
\hline & Subdossel & 0 & , & , & , \\
\hline
\end{tabular}

TABELA 6: Modelos de predição em diâmetro $\left(\operatorname{dap}_{\text {futuro }}=\mathrm{b}_{0}+\mathrm{b}_{1} \mathrm{dap}_{\text {atual }}\right)$ para indivíduos de Araucaria angustifolia, separados por posição sociológica.

TABLE 6: Diameter prediction model $\left(\mathrm{dap}_{\text {futuro }}=\mathrm{b}_{0}+\mathrm{b}_{1} \mathrm{dap}_{\text {atual }}\right)$ for Araucaria angustifolia, separated by social position.

\begin{tabular}{|c|c|c|c|c|c|c|c|c|}
\hline \multirow[b]{2}{*}{ Posição Sociológica } & \multicolumn{4}{|c|}{ Coeficientes } & \multicolumn{4}{|c|}{ Modelo } \\
\hline & Coeficientes & Erro-Padrão & $\mathrm{T}$ & Prob $>t$ & $\mathrm{R}_{\text {ajust }}^{2}$ & $\mathrm{~S}_{\mathrm{yx}} \%$ & $\mathrm{~F}$ & Prob $>$ F \\
\hline \multirow{2}{*}{ Emergente } & $b_{0}=3,271$ & 0,366 & 8,935 & 0,000 & \multirow{2}{*}{0,996} & \multirow{2}{*}{1,699} & \multirow{2}{*}{$31.882,3$} & \multirow{2}{*}{0,000} \\
\hline & $\mathrm{b}_{1}=0,984$ & 0,006 & 178,556 & 0,000 & & & & \\
\hline \multirow{2}{*}{ Intermediária } & $\mathrm{b}_{0}=1,993$ & 0,308 & 6,473 & 0,000 & \multirow{2}{*}{0,992} & \multirow{2}{*}{3,449} & \multirow{2}{*}{$12.488,0$} & \multirow{2}{*}{0,000} \\
\hline & $b_{1}=1,003$ & 0,009 & 111,750 & 0,000 & & & & \\
\hline \multirow{2}{*}{ Subdossel } & $\mathrm{b}_{0}=1,147$ & 0,288 & 3,985 & 0,001 & \multirow{2}{*}{0,995} & \multirow{2}{*}{2,247} & \multirow{2}{*}{$3.238,7$} & \multirow{2}{*}{0,000} \\
\hline & $b_{1}=0,996$ & 0,017 & 56,910 & 0,000 & & & & \\
\hline
\end{tabular}
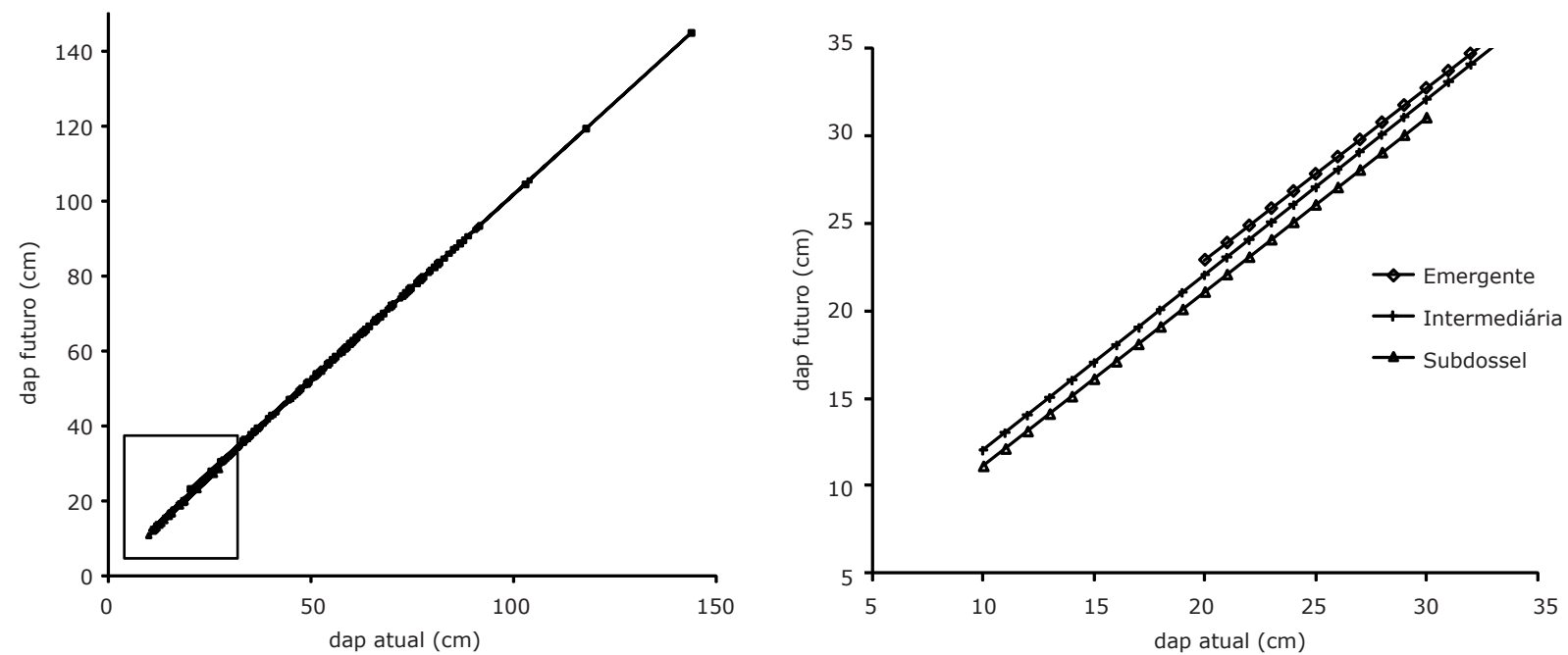

FIGURA 1: Crescimento em diâmetro para as diferentes posições sociológicas da Araucaria angustifolia. FIGURE 1: Diameter growth for different social position of Araucaria angustifolia.

Ci. Fl., v. 21, n. 2, abr.-jun., 2011 
Como os coeficientes $\mathrm{b}_{0}$ e $\mathrm{b}_{1}$ dos modelos de produção estimados para Araucaria angustifolia variaram com a posição sociológica, estes puderam ser reestimados em função dessa variável, sendo:

$$
\begin{gathered}
b_{0}^{\prime}=-1,062 \times P S+4,261, \text { com } R^{2}=0,986 \mathrm{e} \\
b_{1}^{\prime}=-0,013 \times P S^{2}+0,058 \times P S+0,939, \mathrm{com} \\
R^{2}=1,000
\end{gathered}
$$

Juntando essas funções de reestimativa dos coeficientes nas funções de produção anteriormente determinadas, pode-se construir um modelo geral:

$$
\begin{gathered}
d a p_{\text {futuro }}=-1,062 \times P S+4,261+(-0,013 \times \\
\left.P S^{2}+0,058 \times P S+0,939\right) \times d a p_{\text {atual }}
\end{gathered}
$$

Com esse modelo, foi possível determinar o crescimento futuro da espécie, necessitando-se apenas da informação do diâmetro atual e da posição sociológica em que a árvore se encontra.

$\mathrm{Na}$ Figura 2, podem ser observados os erros na estimativa do diâmetro futuro para as posições sociológicas, dos três modelos de produção. $\mathrm{O}$ modelo 1 equivale ao modelo decorrente da análise de covariância, o modelo 2 é decorrente da separação dos indivíduos por posição sociológica e o modelo 3 referente ao modelo geral. Esses erros representam a porcentagem em que o modelo de produção subestimou (valores de erros negativos) ou superestimou (valores de erros positivos) o diâmetro das árvores.

Observou-se que, para as árvores emergentes, os modelos apresentaram a melhor estimativa de diâmetro futuro. Para essa posição sociológica, os erros permaneceram abaixo de $5 \%$, para árvores com diâmetro próximo ou superior a $50 \mathrm{~cm}$. Alguns erros com maior porcentagem podem ser observados para árvores emergentes de menores diâmetros.

Os modelos também apresentaram boas estimativas para árvores subdossel (PS 3), sendo que apenas um indivíduo apresentou um erro maior que $5 \%$.

Já para as árvores intermediárias, os modelos de predição de diâmetro futuro apresentaram maiores porcentagens de erros quando comparados às árvores emergentes e subdossel, sendo alguns desses erros superiores a 5\%. No entanto, para grande maioria dos indivíduos, esses erros ainda permaneceram inferiores a $5 \%$.

Observou-se também que, apesar de os índices de concorrência terem uma alta correlação com o diâmetro, não entraram no modelo de regressão. Isso demonstra que esses índices, da maneira como foram calculados, não conseguem expressar o crescimento e, assim, têm sua utilidade comprometida em uso para modelagens de árvores individuais.
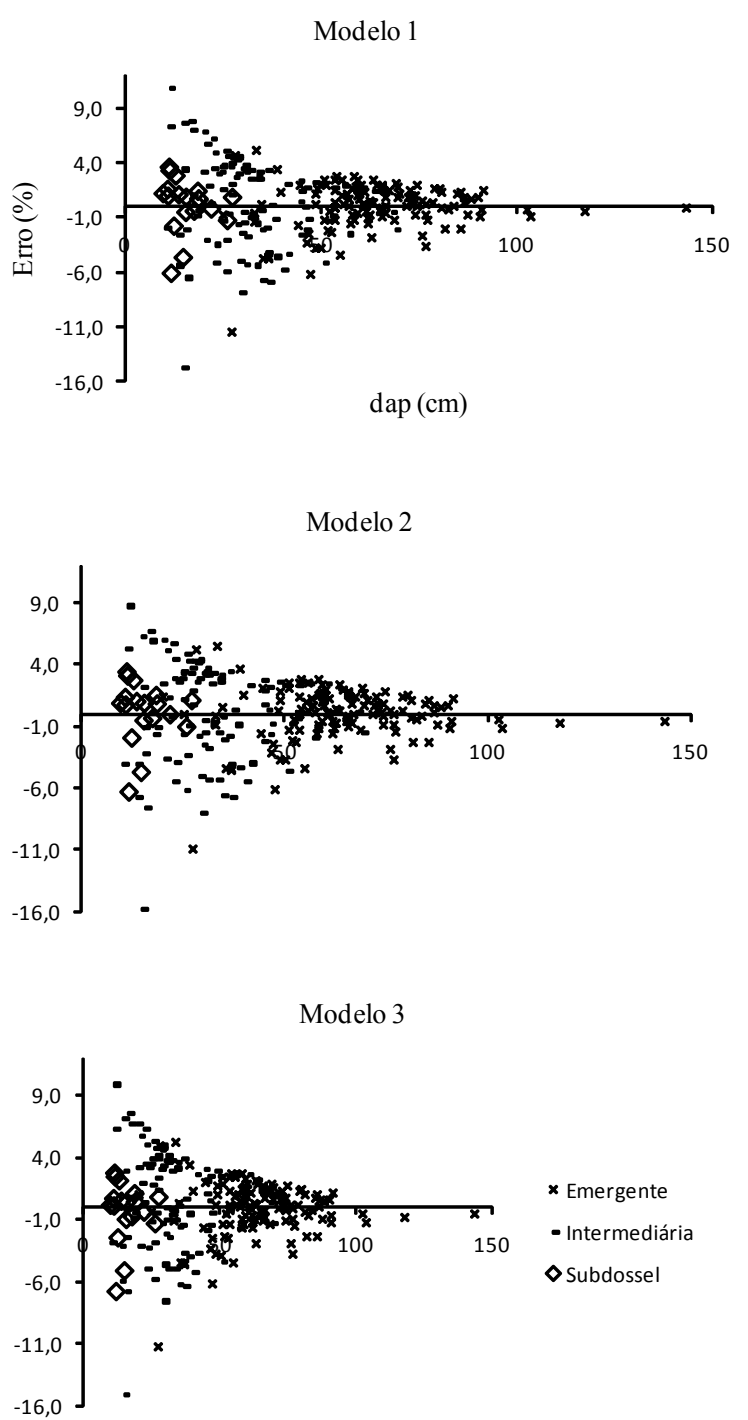

FIGURA 2: Resíduos observados na estimativa do diâmetro futuro de Araucaria angustifolia por meio do modelo de produção, para diferentes posições sociológicas.

FIGURE 2: Observed residual of future diameter estimative of Arancaria angustifolia by yield model, for different social positions. 
Em um trabalho de modelagem realizado para três espécies nativas do Rio Grande do Sul, Cabralea canjerana, Cedrela fissilis e Cordia tricotoma, os índices de concorrência, ao entrarem no modelo, também não aumentaram significativamente o ajuste do modelo (MATTOS, 2007).

Kiernan et al. (2008) modelaram o crescimento em diâmetro para árvores individuais de Acer saccharum em povoamentos mistos de folhosas. Para os autores, os resultados indicaram que os índices de concorrência baseados nas características das árvores individuais não aperfeiçoaram a predição em diâmetro. Somente a variável área basal do povoamento, o diâmetro inicial e a idade após desbaste foram eficientes na estimativa de diâmetro futuro.

\section{CONCLUSÕES}

É possível construir um modelo de crescimento ou produção em diâmetro para árvores individuais de Araucaria angustifolia da Floresta Ombrófila Mista.

A estimativa do diâmetro futuro depende somente do diâmetro atual e da posição sociológica.

Os modelos de produção apresentaram melhores estimativas para árvores emergentes.

Árvores emergentes apresentam maior diâmetro e maior taxa de crescimento que as intermediárias e as subdossel.

Os índices de concorrência independentes de distância, na maneira como foram calculados neste estudo, não mostram eficiência em explicar o crescimento dos indivíduos.

\section{REFERENCIAS BIBLIOGRÁFICAS}

AQUINO, F. M. De. Cultivo de Araucaria angustifolia: análise de viabilidade econômicofinanceiro. Florianópolis: BRDE, 2005. 53 p.

CAMPOS, J. C. C.; LEITE, H. G. Mensuração

Florestal: Perguntas e respostas. 2. ed. Viçosa: UFV, 2006. $470 \mathrm{p}$.

DELLA-FLORA, J. B. Modelos de Crescimento para Árvores Singulares Nectandra megapotamica (Spreng.) Mez. e Ocotea puberula Ness. 2001. 60 f. Dissertação (Mestrado em Engenharia Florestal)Universidade Federal de Santa Maria, Santa Maria, 2001.

ENCINAS, J. I.; SILVA, G. F.; PINTO, J. R. R. Idade e Crescimento das Árvores. Comunicações
Técnicas Florestais, Brasília, v. 7, n. 1, dez. 2005. HASENAUER, H. Ein Einzelbaumwachtumssimulator für ungleichaltrige Fichten-Kiefernund Buchen-Fichtenmischbestände. Fostliche Schriftenreihe, Universität für Bodenkultur, Wien. Band 8, 152 p. 1994.

HESS, A. F.; SCHNEIDER, P. R.; FINGER, C. A. G. Crescimento em diâmetro de Araucaria angustifolia (Bertol.) Kuntze em função da idade, em três regiões do Rio Grande do Sul. Ciência Florestal, Santa Maria, v. 19, n. 1, p. 7-22, jan./mar. 2009.

IBDF/FATEC. Plano de manejo para a Floresta Nacional de São Francisco de Paula - RS. Santa Maria: Ministério do Interior, 1989. 217 p.

KIERMAN, D. H.; BEVILACQUA, E.; NYLAND, R. D. Individual-tree diameter growth model for sugar maple trees in uneven-aged northern hardwood stands under selection system. Forest Ecology and Management, Amsterdam, v. 256, p. 1579-1586, 2008.

LONGHI, S. J. et al. Classificação e caracterização de estágios sucessionais em remanescentes de Floresta Ombrófila Mista na Flona de São Francisco de Paula, RS, Brasil. Ciência Florestal, Santa Maria, v. 16, n. 2, p. 113-125, abri./jun. 2006.

LORENZI, H. Árvores Brasileiras: manual de identificação e cultivo de plantas arbóreas nativas do Brasil. 2. ed.. Nova Odessa, SP: Ed. Plantarum, 1998. 352 p. v. 1

MÄKINEN, H. Effect of Intertree Competition on Branch Characteristics of Pinus sylvestris Families. Canadian Journal Forest Research, Vancouver, v. 11, p. 129-136, 1996.

MATTOS, R. B. Produtividade e incremento de Cabralea canjerana (Vell.) Mart., Cedrela fissilis Vell. e Cordia trichotoma (Vell.) Arrab. Ex Steud., em floresta nativa no Rio Grande do Sul, RS. 2007. 106 f. Tese (Doutorado em Engenharia Florestal)-Universidade Federal de Santa Maria, Santa Maria, 2007.

MORENO, J. A. Clima do Rio Grande do Sul. Porto Alegre: Secretaria da Agricultura, 1961. 41 p. NARVAES, I. S. da. Classificação e caracterização da regeneração em Floresta Ombrófila Mista na Floresta Nacional de São Francisco de Paula, RS. 2004. 134 f. Dissertação (Mestrado em Engenharia Florestal)-Universidade Federal de Santa Maria, Santa Maria, 2004.

NIMER, E. Clima. In: IBGE. Fundação Instituto Brasileiro de Geografia e Estatística. Geografia do Brasil: Região Sul. Rio de Janeiro: 1990, p. 151- 
187.

PORTÉ, A.; BARTELINK, H. H. Modeling mixed forest growth: a review of models for forest management. Ecological Modelling, Amsterdam, v. 150, p. 141-188, 2002.

PRODAN, M. et al. Mensura Forestal 1. San José, C. R.: Deustche Gesellschaft für Tecnische Zusammenarbeit (GTZ) GmbH: Instituto Interamericano de Cooperación para la Agricultura (IICA), 1997. 586 p.

RIBEIRO, S. B. Classificação e ordenação da comunidade arbórea da Floresta Ombrófila Mista da FLONA de São Francisco de Paula, RS. Santa Maria, 2004. 181 f. Tese (Doutorado em Engenharia Florestal) Universidade Federal de Santa Maria, Santa Maria, 2004.

SANQUETTA, C. R. Perspectivas da recuperação e do manejo sustentável das florestas de araucária. Disponível em: $<$ (http:// www.comciencia.br/reportagens/2005/08/09. shtml)> Acesso em: 08/05/09.

SCHNEIDER, P. R.; FINGER, C. A. G.; HOPPE, J. M. Produção de Araucaria angustifolia $\mathrm{O}$. Ktze. na região do planalto médio do estado do Rio Grande do Sul. Ciência Florestal, Santa Maria, v. 2, n. 1, p. 99-118, jan./mar. 1992.

STERBA, H. Forstliche Ertragslehre. Heft 4. Wien: Universität für Bodenkultur, 1992. 160 p.

STRECK, E. V.; et al. Solos do Rio Grande do Sul. 2. ed. Porto Alegre.: EMATER/RS; UFRGS, 2008. $222 \mathrm{p}$.

VANCLAY, J. K. Modelling Forest Growth and Yield: Applications to Mixed Tropical Forest. Wallingford: CAB INTERNATIONAL, 1994, $312 \mathrm{p}$. 